\title{
Noninvasive Assessment of Fetal Pulmonary Blood Flow in Experimental Pulmonary Hypertension in the Fetal Lamb
}

\author{
JEAN-MARIE JOUANNIC, DAMIEN BONNET, ALISON A. HISLOP, RÉGINE ROUSSIN, AND \\ A.-TUAN DINH-XUAN \\ Service de Gynécologie-Obstérique [J.-M.J.], Hôpital Rothschild, AP-HP Paris VI, Paris, France; \\ Cardiopédiatrie [D.B.], Hôpital Necker-Enfants Malades, AP-HP-Université Paris V, 75015 Paris, \\ France; Unit of Vascular Biology and Pharmacology [A.A.H], Institute of Child Health, London, United \\ Kingdom; Département de chirurgie cardiaque pédiatrique [R.R.], Hôpital Marie Lannelongue, 92350 Le \\ plessis Robinson, France; and Service de Physiologie-Explorations Fonctionnelles A.-T.D.-X.], Hôpital \\ Cochin, AP-HP-Université Paris V, 75014 Paris, France
}

\begin{abstract}
ABST
The aim of this study was to assess pulmonary arterial blood
flow changes induced by the creation of a systemic arteriovenous
fistula ( $120 \mathrm{~d}$ gestation) in the fetal lamb using Doppler tech-
nique. Doppler echocardiographic assessment of the pulmonary
artery blood flow performed 1,6 , and $14 \mathrm{~d}$ after surgery showed
that mean pulmonary arterial blood flow in the left or right
pulmonary artery was $224 \pm 58 \mathrm{~mL} /$ min at day 1 in the fistula
group, significantly higher than in the control group (113 \pm 22
mL/min; $p<0.01$, ANOVA test) whether no difference was
found at days 6 and 14 . The mean inner diameter of the left
pulmonary artery measured on postmortem lung arteriograms
compared favorably to the one measured on day 14 at the same
level on ultrasound. The mean left pulmonary arterial blood flow,
measured at birth on day 14 after surgery, using ultrasonic flow
\end{abstract}
During the first minutes of extrauterine life, the pulmonary circulation undergoes marked dilation with a 10 -fold increase of pulmonary arterial flow, allowing the newborn lung to adapt and fulfill its role in gas exchange during postnatal life (1). Failure in this process could lead to persistent pulmonary hypertension of the newborn (PPHN), which is characterized by elevated pulmonary arterial pressure and pulmonary vascular resistance (PVR) leading to severe hypoxemia. A number of perinatal conditions associated with PPHN have been identified, including systemic arteriovenous fistulas.

In an experimental model of systemic fistula in the fetal lamb, we found that at birth there was an increase in pulmonary

Received July 31, 2003; accepted March 29, 2004.

Correspondence: Jean-Marie Jouannic, M.D., Service de Gynécologie-Obstérique, Hôpital Rothschild, AP-HP Paris VI, 33 boulevard de Picpus, Paris, France; E-mail: jean-marie.jouannic@rth.ap-hop-paris.fr

This work was supported by a grant from Fondation de l'Avenir, Paris, France. A.A.H. is supported by the British Heart Foundation.

DOI: 10.1203/01.PDR.0000136286.65985.7C transducer, was not statistically different from the one measured by Doppler on day 14. Our data demonstrate that echocardiography allows accurate assessment of pulmonary arterial blood flow in utero, providing evidence suggesting transient high pulmonary blood flow that did not last $>6 \mathrm{~d}$ after the creation of a systemic fistula. (Pediatr Res 56: 385-390, 2004)
Abbreviations
DA, ductus arteriosus
FHR, fetal heart rate
LPA, left pulmonary artery
PPHN, persistent pulmonary hypertension of newborn
RPA, right pulmonary artery

arterial pressure and resistance with no increase in pulmonary arterial blood flow (2). This was associated with structural remodeling of the pulmonary vascular bed similar to that described in neonates with PPHN (3). In the model of prenatal ductus arteriosus (DA) compression, in which a temporary increase in pulmonary arterial blood flow was found, similar remodeling of the fetal pulmonary arteries has been described $(4,5)$. This led us to hypothesize that a systemic arteriovenous fistula by increasing blood volume returning to the right heart may lead to temporary pulmonary high blood flow in utero.

Recent improvement in high-resolution ultrasound equipment, including color Doppler, has allowed more accurate assessment of fetal blood flow distribution. Doppler echocardiographic studies in the human fetuses have focused on the cardiac output and the distribution of blood flow to the pulmonary and systemic circulation in physiologic conditions (6, 7). In this study, we aimed to assess pulmonary arterial blood flow changes induced by the creation of a systemic arteriovenous fistula in the fetal sheep using a noninvasive Doppler 
technique and also to relate the measurements made on the images achieved to the postmortem structure of the pulmonary artery.

\section{METHODS}

Surgical preparation. Twenty fetal lambs were obtained from 18 pregnant PreAlp ewes and divided into two groups. This study was approved by the ethical committee of our institution, and animals received care in accordance with the "Principles of Laboratory Animal Care" and the "Guide for the Care and Use of Laboratory Animals" (8). In nine fetal lambs, a systemic arteriovenous fistula was created at $120 \mathrm{~d}$ of gestation (full-term $145 \mathrm{~d}$ ). The pregnant ewes were fasted for $48 \mathrm{~h}$ before surgery. Ewes were sedated with i.v. injection of pentothal $(10 \mathrm{mg} / \mathrm{kg}$ of body weight) and anesthetized with $1 \%$ Halothane. The surgical technique for creating the fistula between the jugular vein and the carotid artery has been described previously (2). Antibiotics ( $1 \mathrm{~g}$ of ampicillin and $50 \mathrm{mg}$ of gentamicin sulfate) were administrated to the ewe during surgery and repeated daily for $5 \mathrm{~d}$.

The control group of 11 fetuses comprised 7 fetuses from unoperated ewes, 2 unoperated fetuses whose co-twin was operated on, and 2 sham-operated fetuses that were subjected to the surgical procedure, but the fetal jugular vein and the carotid artery were only visualized and no anastomosis was performed.

In vivo Doppler study. Doppler echocardiographic assessment of the pulmonary artery and aortic blood flow was performed 1,6 , and $14 \mathrm{~d}$ after surgery by the same investigator (J.-M.J.). The ewes were fasted $12 \mathrm{~h}$ before the procedure. Maternal general anesthesia was induced by $400 \mathrm{mg}$ of ketamine and $15 \mathrm{mg}$ of diazepam (i.v.) and maintained by $150 \mathrm{mg}$ of ketamine every $10 \mathrm{~min}$ and $10 \mathrm{mg}$ of diazepam every $30-60$ min (9). Maternal heart rate and saturation of arterial oxygen were monitored continuously. In all cases, high-resolution color Doppler ultrasound equipment with 5- to 3- and 7- to 4-MHz broadband transducers and spatial peak temporal average intensities $<100 \mathrm{~mW} / \mathrm{cm}^{2}$ were used. The fetal lamb was visualized by a transabdominal scan with the ewe in the supine position.

Views of the left pulmonary artery (LPA) and right pulmonary artery (RPA) were obtained from a short-axis view and an oblique transverse plane of the fetal chest, using color Doppler imaging (7). Depending on the fetal position, the velocity waveforms from the LPA or the RPA were studied. The descending aorta was visualized on a sagittal plane, and the velocity waveforms were recorded at the lower thoracic level just above the diaphragm (10). The inner diameter of the vessel was determined during systole by averaging three measurements with an insonation perpendicular to the long axis (6). The blood velocity was recorded with pulsed Doppler at the lowest possible angle of interrogation. Angle correction was not used. The sample volume was set at 1 to $3 \mathrm{~mm}$ and positioned in the center of the vessel. The high-pass filter was set at 100 to $200 \mathrm{~Hz}$. At least three consecutive uniform Doppler velocity waveforms were recorded on hard copy, and one cycle was analyzed using a microcomputer. In each case,
LPA or RPA and aortic blood flows were calculated using the formula Blood Flow $=\left(\right.$ TVI $\left.\cdot \pi \cdot \mathrm{d}^{2} / 4\right) \mathrm{FHR}$, where TVI is time velocity integral, $d$ is the vessel diameter, and FHR is fetal heart rate.

Delivery and physiologic measurements. On day 134, after the 14-d Doppler assessment, ewes underwent a second laparotomy under the same anesthetic procedures. The fetal chest was exposed through a uterine incision. A left thoracotomy exposed the heart and great vessels. Polyvinyl catheters were inserted and secured into aorta, left atrium, and LPA by direct puncture after the placement of purse string sutures (2). An ultrasonic flow transducer size 6 (Transonic systems, Ithaca, NY, U.S.A.) was placed around the LPA to measure blood flow (3). All measurements were made after a 10 -min recovery period. Mean pressures were obtained by electrical integration and recorded on a multichannel recorder (Sirem Siemens, Erlangen, Germany). Pulmonary vascular resistance was calculated as the difference between mean LPA and left atrial pressure divided by flow (4). After measurements, the fistula patency was confirmed and lambs were killed by quick exsanguination. The lambs were weighed. The heart and lungs were removed en bloc immediately after death and weighed.

Lung arteriograms. Lungs were separated from the heart and stored overnight at $4^{\circ} \mathrm{C}$. The following day, they were brought back to room temperature for $2 \mathrm{~h}$. A catheter was then inserted into the main pulmonary artery and secured with a ligature. The pulmonary arteries were injected at a pressure of $40 \mathrm{~mm} \mathrm{Hg}$, which is similar to the physiologic pressures found in control animals (3) with a warm barium-gelatin mixture at $60^{\circ} \mathrm{C}(500 \mathrm{~mL}$ of distilled water, $200 \mathrm{~mL}$ of Micropaque powder, $50 \mathrm{~g}$ of gelatin Bloom 8-G) as previously described (11). To obtain comparable measurements on the left pulmonary arteries, we used the same pressure for both groups. For each lamb, the lumen diameters of the LPAs were measured on the arteriograms at four levels by the same investigator (J.M.J.) (Fig. 1).

Statistical analysis. Data were expressed as mean \pm SD. The hemodynamic data were analyzed using $t$ test. Comparisons between the two groups were made by two-way ANOVA. When statistical differences were identified, a post hoc analysis with Fisher's protected least significant difference test was performed.

For assessing the agreement between 1) Doppler assessment and direct measurement of the pulmonary artery blood flow and 2) between ultrasound and postmortem measurement of the internal diameter of the LPA at day 14, the difference between the measurements of the two methods was plotted against their mean using the method of Bland and Altman (12). The normal distribution of the differences was verified by the normal method plot (13). Systematic bias between the two methods was analyzed by ANOVA. Limits of agreement and 95\% confidence intervals were calculated as previously described $(12,13)$.

\section{RESULTS}

Of the nine fetuses in which a fistula was created, seven were alive at the time of the elective cesarean section; two 


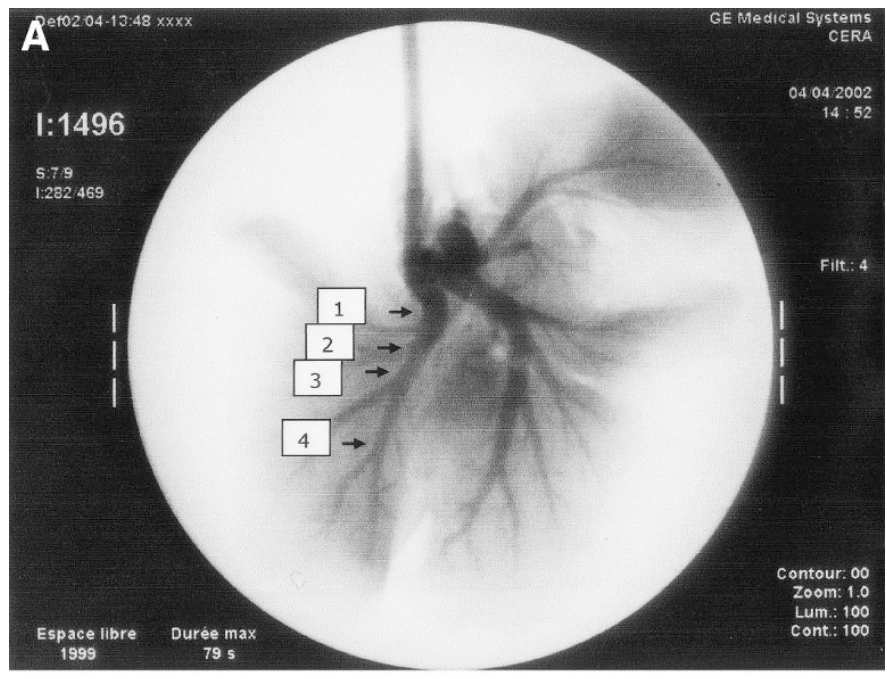

Table 1. In vivo Doppler assessment of the pulmonary and aortic blood flow

\begin{tabular}{lll}
\hline & Control & Fistula \\
\hline FHR (bpm) & & \\
$\quad$ Day 1 & $178 \pm 35(11)$ & $175 \pm 15(9)$ \\
Day 6 & $158 \pm 18(10)$ & $153 \pm 8(7)$ \\
Day 14 & $162 \pm 45(10)$ & $148 \pm 14(7)$ \\
Pulmonary blood flow (mL/min) & & \\
$\quad$ Day 1 & $113 \pm 22(11)$ & $224 \pm 58^{*}(9)$ \\
Day 6 & $118 \pm 9(10)$ & $137 \pm 37(7)$ \\
Day 14 & $124 \pm 24(10)$ & $122 \pm 15(7)$ \\
Aortic blood flow (mL/min) & & \\
Day 1 & $429 \pm 83(11)$ & $642 \pm 114 \dagger(9)$ \\
Day 6 & $463 \pm 79(10)$ & $557 \pm 82(7)$ \\
Day 14 & $487 \pm 71(10)$ & $474 \pm 72(7)$ \\
\hline
\end{tabular}

Values are expressed as mean $\pm \mathrm{SD}$. The number of animals is indicated in brackets.

$* p<0.01, \dagger p<0.05$, ANOVA test.
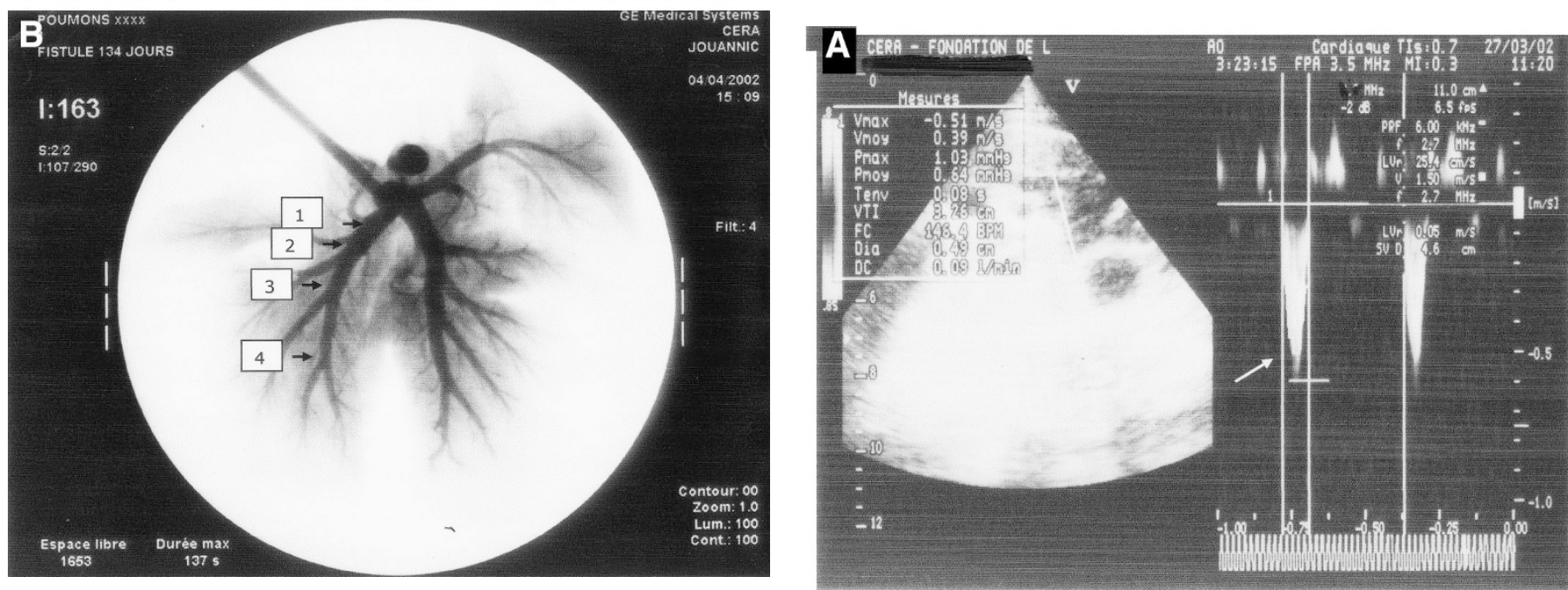

Figure 1. Postmortem lung arteriograms. $(A)$ Control animal. (B) Animal from the fistula group. The arrows indicate the levels (1-4) at which the lumen diameters of the LPAs were measured. Level 1 corresponds to the level studied for echocardiographic study.

stillborn animals were delivered vaginally 3 and $5 \mathrm{~d}$ after surgery. Postmortem examination did not reveal any sign of hydrops or infection. In the control group, 10 fetuses were alive at the time of delivery. One sham-operated fetus was stillborn at $3 \mathrm{~d}$ after surgery with no abnormality on postmortem examination. The Doppler data at day 1 from these three stillborn fetuses were available, and they were included in the analysis.

In vivo Doppler study. The fistula did not induce any significant change of the FHR between the two groups (Table 1). Technically acceptable pulmonary and aortic artery flow velocity waveforms with well-defined systolic and diastolic components were obtained in all cases. The calculated mean pulmonary arterial blood flow in the LPA or the RPA was $224 \pm 58 \mathrm{~mL} / \mathrm{min}$ at $1 \mathrm{~d}$ after surgery in the fistula group, significantly higher than in the control group $(113 \pm 22$ $\mathrm{mL} / \mathrm{min} ; p<0.01$, ANOVA test; Fig. 2). There was no difference for the calculated LPA or RPA blood flow between the two groups at day 6 or day 14 (Table 1). For assessing the initial change of the arterial pulmonary blood induced by the

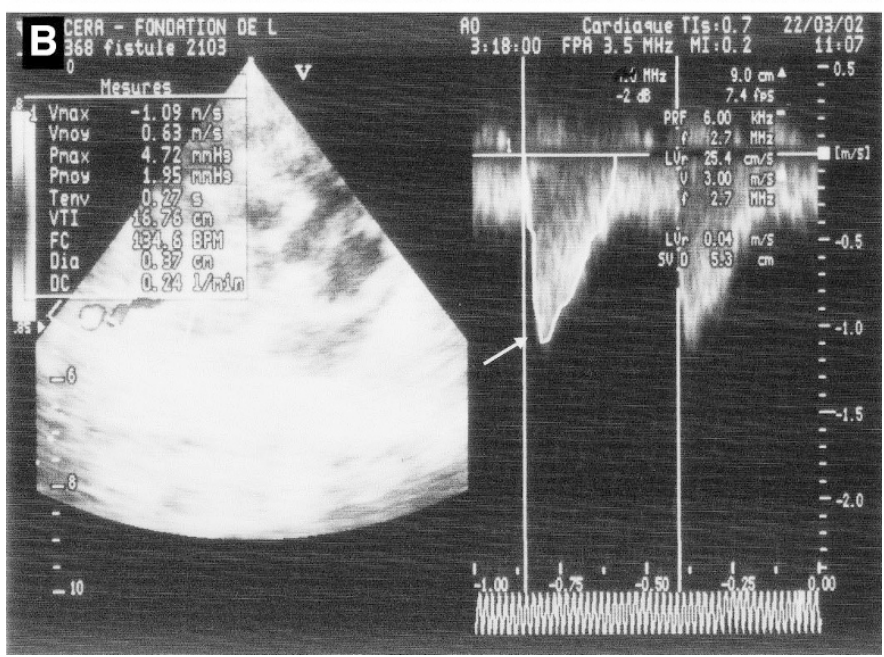

Figure 2. Doppler velocity waveforms of blood flow in the LPA measured at day 1 after surgery. (A) Control fetus: inner diameter of the vessel, $4.9 \mathrm{~mm}$; LPA blood flow, $90 \mathrm{~mL} / \mathrm{min}$. (B) Fetus with arteriovenous fistula: inner diameter of the vessel, $3.7 \mathrm{~mm}$; LPA blood flow, $240 \mathrm{~mL} / \mathrm{min}$. The arrows indicate the pulsed wave velocity spectrum in the LPA.

fistula, pulmonary blood flow was also determined by Doppler 30 min after surgery in two fetuses. LPA or RPA blood flow was 170 and $190 \mathrm{~mL} / \mathrm{min}$, respectively, which was similar to 
the measurements made on day 1 on the same animals (180 and $185 \mathrm{~mL} / \mathrm{min}$, respectively). The calculated mean blood flow in the descending aorta was higher in the fistula group $1 \mathrm{~d}$ after surgery ( $p<0.05$, ANOVA test; Table 1$)$, but no significant difference was observed at days 6 and 14 between the two groups.

Mean inner diameter of the pulmonary arteries measured on ultrasound was higher in the fistula group at all time points studied but was not statistically different (Table 2). Inner diameters of the RPA or LPA were not different in both groups.

In vivo physiologic study. The mean LPA pressure was $48 \pm 2$ in the fistula group, significantly higher than in the control group (39 $\pm 2 \mathrm{~mm} \mathrm{Hg} ; p<0.05, t$ test). The mean aortic pressure and left atrial pressure were not different between the two groups. There was no difference for the LPA blood flow measured using ultrasonic flow transducer between the two groups (fistula group: $138.5 \pm 20 \mathrm{~mL} / \mathrm{min}$; control group: $142.3 \pm 16 \mathrm{~mL} / \mathrm{min}$ ). In the fistula group, the pulmonary vascular resistance was $0.38 \mathrm{~mm} \mathrm{Hg} \cdot \mathrm{mL}^{-1} \cdot \min ^{-1}$, significantly higher than in the control group $(0.26 \mathrm{~mm} \mathrm{Hg}$. $\left.\mathrm{mL}^{-1} \cdot \min ^{-1} ; p<0.05\right)$.

All of the Doppler measurements followed a normal distribution according to the normal plot method (data not shown). Mean difference between LPA blood flow measured by ultrasound and LPA blood flow measured using the ultrasonic flow transducer was $-7.05 \mathrm{~mL} / \mathrm{min}$, and the $95 \%$ confidence interval was -9.53 to $-4.59 \mathrm{~mL} / \mathrm{min}$, using the Bland-Altman method of comparison. Although not statistically significant ( $p$ $=0.65$, ANOVA test), the Doppler measurement tends to underestimate the pulmonary blood flow. However, as shown in Fig. 3A, 16 (95\%) of 17 of the measurements were within the limits of agreement.

Postmortem studies. The mean fetal weight and mean lung weight were not different in the two groups (fistula group: fetal weight, $2535 \pm 135 \mathrm{~g}$, lung weight, $128 \pm 12 \mathrm{~g}$; control group: fetal weight, $2485 \pm 150 \mathrm{~g}$, lung weight, $124 \pm 8 \mathrm{~g}$ ).

Lung arteriograms of all lambs showed a uniform vascular filling, and no difference was found between the two groups in the pulmonary arterial branching pattern (Fig. 3). The mean left lung height measured on arteriograms was not different between the two groups (fistula group: $76 \pm 7 \mathrm{~mm}$; control group: $79 \pm 9 \mathrm{~mm}$ ). No difference was found in the mean inner diameter of the left pulmonary artery measured at the four levels between the groups (Table 3). The mean difference between the inner diameter of the LPA measured by ultrasound and the one measured at level 1 on postmortem arteriograms was $-0.1 \mathrm{~mm}$, and the $95 \%$ confidence interval was -0.18 to $-0.01 \mathrm{~mm}$ (Fig. $3 B$ ).

Table 2. Internal diameter of the extrapulmonary artery measured

\begin{tabular}{lccc}
\multicolumn{4}{c}{ on ultrasound } \\
\hline & Control & Fistula & $p$ value* \\
\hline Day 1 & $3.9 \pm 0.3(11)$ & $4.3 \pm 0.5(9)$ & 0.45 \\
Day 6 & $4.7 \pm 0.4(10)$ & $4.8 \pm 0.4(7)$ & 0.87 \\
Day 14 & $4.4 \pm 0.5(10)$ & $4.7 \pm 0.5(7)$ & 0.76 \\
\hline
\end{tabular}

Values are in millimeters and expressed as mean \pm SD. The number of animals is indicated in brackets.

* ANOVA test.

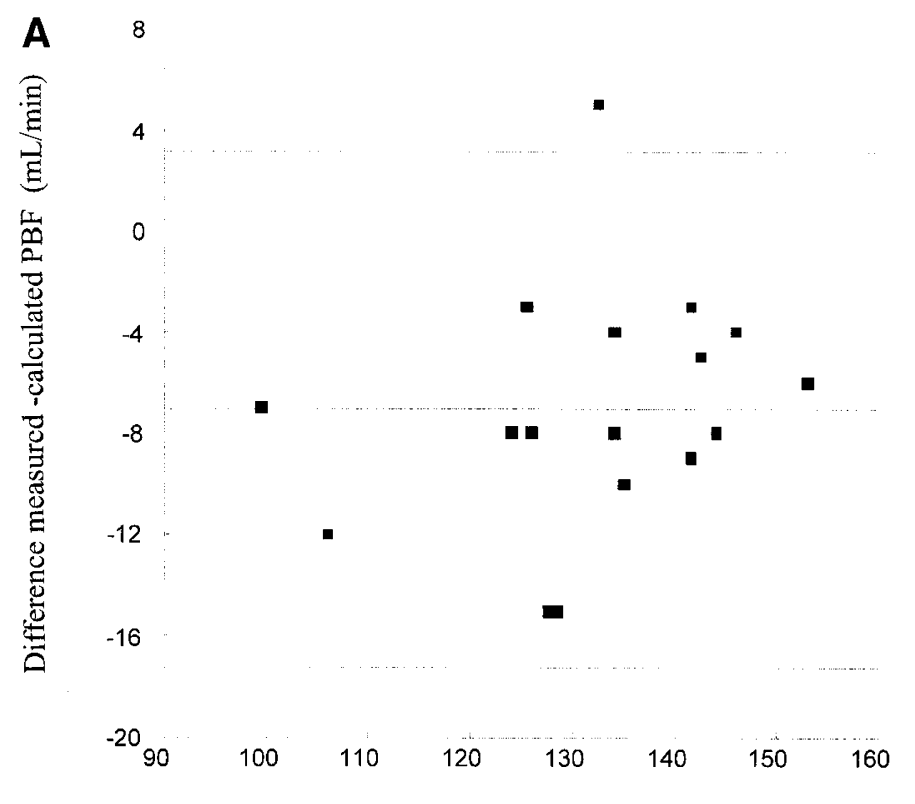

Mean pulmonary blood flow $(\mathrm{mL} / \mathrm{min})$

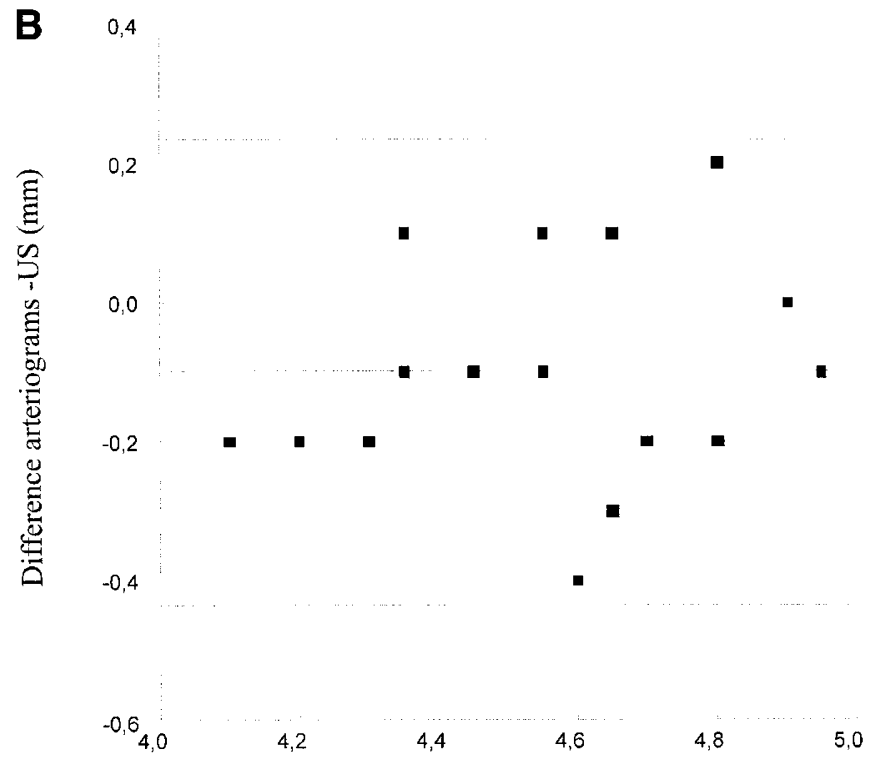

Mean internal diameter of the extrapulmonary artery $(\mathrm{mm})$

Figure 3. Differences plotted against their mean for Doppler assessment and direct measurement of the pulmonary artery blood flow $(r=0.93, p<0.0001$; $A$ ) and between ultrasound and postmortem measurement of the internal diameter of the LPA at day $14(r=0.83, p<0.0001 ; B)$. Mean differences and the limits of agreement are shown in continuous lines. PBF, pulmonary blood flow; US, ultrasound.

\section{DISCUSSION}

Our data demonstrate that the creation of a systemic arteriovenous fistula in the fetal lamb is associated with a temporary high pulmonary blood flow. Mean pulmonary arterial blood flow was twice as high on day 1 in the fistula group than in controls, whereas by 6 or $14 \mathrm{~d}$ after surgery, no difference was found between the two groups. This study is the first application of Doppler echocardiographic blood flow assessment to the pulmonary artery branch in a prenatal model of 
Table 3. Internal diameter of the LPA measured at four levels on postmortem arteriogram

\begin{tabular}{rrrc}
\hline & \multicolumn{1}{c}{$\begin{array}{c}\text { Control } \\
(n=10)\end{array}$} & $\begin{array}{c}\text { Fistula } \\
(n=7)\end{array}$ & $p$ value* \\
\hline Level 1 & $4.5 \pm 0.5$ & $4.9 \pm 0.3$ & 0.60 \\
Level 2 & $4 \pm 0.5$ & $3.8 \pm 0.4$ & 0.74 \\
Level 3 & $3.2 \pm 0.3$ & $3.4 \pm 0.2$ & 0.86 \\
Level 4 & $2 \pm 0.2$ & $2 \pm 0.2$ & 0.95 \\
\hline
\end{tabular}

Values are in millimeters and expressed as mean $\pm \mathrm{SD}$.

* ANOVA test.

pulmonary hypertension and confirms that the estimated blood flow and size of the pulmonary artery were accurate.

Most of the current knowledge on fetal pulmonary blood flow was derived from invasive techniques using either radionucleotide-labeled microspheres $(1,14)$ or more recently electromagnetic flow transducers (4). Although quantification of blood flow using electromagnetic flowmeter is considered the gold standard, this method has a reported error of $\sim 10 \%$ related to inaccuracy in measuring volume and time intervals (15). In addition, the application of an electromagnetic flow transducer is limited to animal studies because of its invasive character.

Doppler echocardiographic blood flow determination has been validated in both in vitro experiments and animal models $(7,16)$. More recently, Doppler echocardiographic studies in the human fetuses have focused on the cardiac output and the distribution of blood flow to the pulmonary and the systemic circulations in physiologic conditions $(6,17,18)$. These studies clarified the classical concepts on fetal hemodynamics that were derived from animal invasive studies, and such studies may not be correct for the human fetus (6).

Although the Doppler technique has been found to quantify blood flow accurately in several animal models, some of the limitations of this technique are worthy of mention. Errors in volumetric blood flow calculation may arise from the determination of both vessel size and the Doppler waveforms. In our study, following the recommendation of previous studies, the internal vessel diameter was measured according to the trailing to leading-edge method (i.e. from the inner edge of the anterior vessel wall to the inner edge of the posterior vessel wall) $(6,7)$. This method prevents the risk of overestimating the vessel area and, as a consequence, the blood flow. Because of the relatively small internal diameter of the pulmonary artery branch, any inaccuracy in the internal vessel diameter determination is likely to be a major source of error in blood flow calculation. Improvement of ultrasound equipment with high axial resolution makes it possible to measure precisely the internal diameter of small vessels. In the present study, the inner diameter of the pulmonary arteries measured on ultrasound on day 14 after surgery were consistent with those measured on postmortem arteriograms.

Inaccuracy in velocity determination represents another major source of error in blood flow determination (16). Angle correction is essential for flow velocity determination. To avoid the influence of insonation angle, we studied the pulmonary branch (either right or left) that allowed an angle of $<30$ degrees. In addition, the use of color Doppler imaging allowed the pulse-wave Doppler sample volume to be positioned pre- cisely in the middle of the vessel at a distance from the main pulmonary artery bifurcation, consistently $>15 \mathrm{~mm}$. This was necessary to avoid any effect of disturbed flow that is often present near the bifurcation point of a vessel (19). Finally, the anesthetic protocol that we used allowed the blood velocity to be measured in the absence of fetal movement, preventing an off-axis sampling error. We found that Doppler assessment tends to underestimate pulmonary blood flow as compared with the standard method of measurement using electromagnetic flow transducer. However, we believe that the mean difference together with the $95 \%$ confidence interval were small enough to make the Doppler assessment acceptable for clinical applications. As poor interobserver reproducibility of Doppler estimates of volume flow have been reported in human fetuses (20, $21)$, it is advisable to perform sequential measurements by one single operator, as recommended by Simpson et al. (20).

The initial increase in pulmonary arterial blood flow after the creation of the fistula was not associated with a significant increase in the internal diameter on the pulmonary arteries measured on ultrasound. Whether a subtle change in the internal diameter of the pulmonary arteries, sufficient to allow a significant increase in flow, is amenable to ultrasound measurement remains to be determined. The temporary increase in pulmonary arterial blood flow that we observed may reflect a temporary decrease in the pulmonary arterial resistance similar to that reported in the DA compression model (5). Postmortem pulmonary arteriograms did not reveal any significant increase in the internal diameter of the pulmonary arteries in the fistula group. This is consistent with our previous report on the size of the intralobar arteries, conduit or peripheral, that showed no difference between the two groups (3). We found that the structural remodeling of an increase in smooth muscle affected only peripheral vessels, and these might play a key role in time-dependent changes of the pulmonary arterial blood flow observed in our model.

The $50 \%$ increase in the pulmonary arterial blood flow found $1 \mathrm{~d}$ after surgery was comparable with the one reported by Abman et al. (5) in the model of prenatal DA compression using an electromagnetic flowmeter. However, the temporary pulmonary high blood flow derived from our Doppler study seemed to be more sustained compared with the one reported by Abman, in which the LPA blood flow returned to baseline value $2-3 \mathrm{~h}$ after the DA compression, whereas LPA pressure and resistance remained high. In an additional study by this group, the normalization of pulmonary blood flow was found to be related to myogenic response (22). We found that the surgical creation of the fistula was associated with a rapid increase of the pulmonary blood flow (in the first $30 \mathrm{~min} ; n=$ 2 fetuses) and that this high blood flow was sustained for at least $24 \mathrm{~h}$. This delay in the normalization of pulmonary blood flow may reflect a less pronounced or delayed myogenic response in the fistula model. Further in vivo studies should focus on the mechanism responsible for this apparent delay in normalization of the arterial pulmonary blood flow in the fistula model.

In conclusion, we report that the prenatal creation of a systemic arteriovenous fistula in the fetal lamb is associated with a temporary high pulmonary blood flow. This study 
demonstrates the feasibility of Doppler-derived pulmonary blood flow assessment. The Doppler techniques that we used are the same as those commonly used for human fetal hemodynamic evaluation in clinical practice. Our results should encourage the assessment of fetal pulmonary arterial blood flow in prenatal pathophysiologic conditions.

\section{REFERENCES}

1. Rudolph AM 1985 Distribution and regulation of blood flow in the fetal and neonatal lamb. Circ Res 57:811-821

2. Jouannic JM, Martinovic J, Roussin R, Laborde F, Dumez Y, Dinh-Xuan AT 2002 The effect of a systemic arteriovenous fistula on the pulmonary arterial blood pressure of the fetal sheep. Prenat Diagn 22:48-51

3. Jouannic JM, Roussin R, Hislop AA, Lanone S, Martinovic J, Boczkowski J, Dumez Y, Boczkowski J, Dinh-Xuan AT 2003 Systemic arteriovenous fistula leads to pulmonary artery remodeling and abnormal pulmonary vasoreactivity in fetal lamb. Am J Physiol Lung Cell Mol Physiol 285:L701-L709

4. Abman SH, Shanley PF, Accurso FJ 1989 Failure of postnatal adaptation of the pulmonary circulation after chronic intrauterine pulmonary hypertension in the fetal lambs. J Clin Invest 83:1849-1858

5. Abman SH, Accurso FJ 1989 Acute effects of partial compression of ductus arteriosus on fetal pulmonary circulation. Am J Physiol Heart Circ Physiol 257:H626-H634

6. Mielke G, Benda N 2001 Cardiac output and central distribution of blood flow in the human fetus. Circulation 103:1662-1668

7. Stewart WJ, Jiang L, Mich R, Pandian N, Guerrero JL, Weyman AE 1985 Variable effects of changes in flow rate though the aortic, pulmonary and mitral valves on valve area flow velocity: impact on quantitative Doppler flow calculations. J Am Coll Cardiol 6:653-662

8. Bayne K 1998 Developing guidelines on the care and use of animals. Ann N Y Acad Sci 862:105-110
9. Kiserud T, Ozaki T, Nishina H, Rodeck C, Hanson MA 2000 Effect of NO, phenylephrine, and hypoxemia on ductus venosus diameter in fetal sheep. Am J Physiol Heart Circ Physiol 279:H1166-H1171

10. Jouannic JM, Benachi A, Bonnet D, Fermont L, Dumez Y, Le Bidois J, Dommergues M 2002 Middle cerebral artery Doppler in fetuses with transposition of the great arteries. Ultrasound Obstet Gynecol 20:122-124

11. Reid LM 1979 The pulmonary circulation: remodeling in growth and disease. The 1978 J. Burns Amberson lecture. Am Rev Respir Dis 119:531-546

12. Bland JM, Altman DG 1986 Statistical methods for assessing agreement between two methods of clinical measurement. Lancet 1:307-310

13. Bland JM, Altman DG 2003 Applying the right statistics: analyses of measurement studies. Ultrasound Obstet Gynecol 22:85-93

14. Morin FC 3rd, Egan EA 1989 The effect of closing the ductus arteriosus on the pulmonary circulation of the fetal sheep. J Dev Physiol 11:283-287

15. Zierler BK, Kirkman TR, Kraiss LW, Reiss WG, Horn JR, Bauer LA, Clowes AW, Kholer TR 1992 Accuracy of duplex scanning for measurement of arterial volume flow. J Vasc Surg 16:520-526

16. Gill RW 1985 Measurement of blood flow by ultrasound: accuracy and sources of error. Ultrasound Med Biol 11:625-641

17. Mielke G, Benda N 2000 Blood flow velocity waveforms of the fetal pulmonary artery and the ductus arteriosus: reference ranges from 13 weeks to term. Ultrasound Obstet Gynecol 15:213-218

18. Sutton MS, Groves A, MacNeill A, Sharland G, Allan L 1994 Assessment of changes in blood flow through the lungs and foramen ovale in the normal human fetus with gestational age: a prospective Doppler echocardiographic study. Br Heart J 71:232237

19. Ku DN, Giddens DP, Phillips DJ, Strandness DE Jr 1985 Hemodynamics of the normal human carotid bifurcation: in vitro and in vivo studies. Ultrasound Med Biol $11: 13-26$

20. Simpson JM, Cook A 2002 Repeatability of echocardiographic measurements in the human fetus. Ultrasound Obstet Gynecol 20:332-339

21. Brodszki J, Gardiner HM, Eriksson A, Stale H, Marsal K 1998 Reproducibility of ultrasonic fetal volume blood flow measurements. Clin Physiol 18:479-485

22. Storme L, Rairigh RL, Parker TA, Kinsella JP, Abman SH 1999 In vivo evidence for a myogenic response in the fetal pulmonary circulation. Pediatr Res 45:425-431 